\title{
A Dual Sono/Photochemical Fabrication of the Sage Leaves Extract Coated Silver Nanoparticles
}

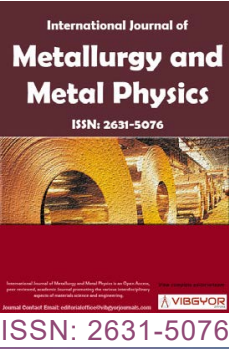

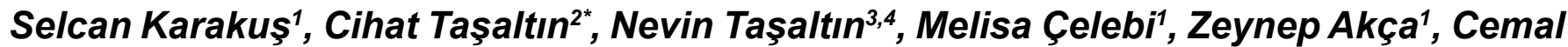 Özeroğlu ${ }^{1}$ and Ayben Kilislioğlu ${ }^{5}$}

${ }^{1}$ Department of Chemistry, Istanbul University-Cerrahpaşa, Turkey

${ }^{2}$ TUBITAK Marmara Research Center, Materials Institute, Turkey

${ }^{3}$ Department of Electrical-Electronics Engineering, Maltepe University, Turkey

${ }^{4}$ Department of Renewable Energy Tech., Maltepe University, Turkey

${ }^{5}$ Department of Electrical-Electronics Engineering Istanbul, Kadir Has University, Turkey

\begin{abstract}
In this study, we prepared the biodegradable sage leaves extract coated silver nanoparticles (SLE-AgNPs) using a green dual sono/photochemical synthesis route. The SLE-AgNPs were successfully synthesized from silver nitrate using the SLE as a green reducing and stabilizing agent. The morphological characterization and functional groups of SLE and SLE-AgNPs were determined by different techniques such as scanning electron microscopy (SEM) with energy-dispersive X-ray (EDX) analyzer, transmission electron microscope (TEM), Fourier transform infrared (FTIR) spectroscopy, Brunauer-Emmett-Teller (BET), dynamic light scattering (DLS), and X-ray diffraction (XRD). We examined the effects of experimental parameters such as the amplitude, sonication time, and temperature on the rheological results. According to the obtained experimental results, we proved that the SLE NPs had a uniform spherical shape under the optimum experimental conditions. SLE-AgNPs were obtained with high colloidal stability. The average diameter range of SLE-AgNPs was $\sim 120 \mathrm{~nm}$ while the surface area determined by the BET measurement was 150.67 $\mathrm{m} 2 / \mathrm{g}$. Furthermore, we performed to evaluate the accuracy of the experimental results obtained error analysis using surface tension and conducted the error analysis to assess the accuracy of the experimental results using the surface tension and process parameters. Consequently, we proved that the SLE-AgNPs can be successfully synthesized using the ultrasonic method and the synthesized AgNPs can be a promising nanocarrier in multiple applications for the future.
\end{abstract}

\section{Keywords}

Sage leaves, Silver nanoparticles, Dual sono/photochemical synthesis, Ultrasonic method

\section{Introduction}

Nanomaterials have been attracted scientists in various application areas such as textile, sensor, energy, antibacterial, and drug delivery system due to their superior chemical, biological, optical and mechanical properties [1-3]. It ensures that nano-

*Corresponding author: Cihat Taşaltın, TUBITAK Marmara Research Center, Materials Institute, Gebze, Kocaeli, Turkey

Accepted: May 01, 2021; Published: May 03, 2021

Copyright: (C) 2021 Karakuş S, et al. This is an open-access article distributed under the terms of the Creative Commons Attribution License, which permits unrestricted use, distribution, and reproduction in any medium, provided the original author and source are credited.

Karakuş et al. Int J Metall Met Phys 2021, 6:067

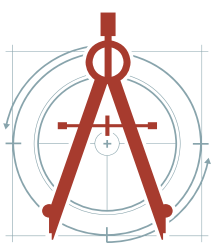


materials have superior properties compared to conventional materials, depending on their size, shape, particle distribution, chemical composition, stability, surface area, and surface properties with more reactivity towards chemicals $[4,5]$. In recent years, many studies have been reported for the preparation and biomedical applications of metal based nanoparticles such as gold, silver, titanium, and copper [6-10]. Among these nanostructures, silver nanoparticles that have antibacterial properties have been widely studied for their superior physicochemical, biological, optical, and mechanical properties [11-15].

Silver nanoparticles have been reported to be synthesized by different methods such as microwave-assisted method [16], laser ablation method [17], hydrothermal synthesis [18] high energy ball milling [19], chemical reduction method [20], electrochemical synthesis [21], biomimetic synthesis [22], ultraviolet-visible light irradiation [23], and ultrasonic-assisted method [24]. While fabricating silver nanoparticles, most of the methods have some disadvantages, such as the use of toxic volatile organic solvents, high temperature and pressure requirements, toxic by-products, and high-cost [25].

In this study, considering the current disadvantages, we aimed to synthesize the sage leaves extract coated with silver nanoparticles (SLE-AgNPs) by the ultrasonic method in a short time ( $5 \mathrm{~min}$ ), economically, without the need for high temperature and pressure with the green chemistry approach [26]. Considering these environmental concerns, the aim of this study is to develop the preparation of SLE-AgNPs with homogeneous distribution by a fast method under high-intensity ultrasonic irradiation based on green chemistry principles. There have been several studies focused on the different bio-materials based matrix such as acalypha fruticosa leaf extracts [27], pergularia daemia leaf extract [28], plant leaf [29], brown algae [30], and green tea [31] extracts acted as a reducing and stabilizing agent to obtain the AgNPs. The functional groups such as ketone, aldehyde, amide, terpenoid, flavone and carboxylic acid in the biomaterial extracts, which have excellent biocompatibility and biodegradability properties and play a dual role in the reduction and stabilization of $\mathrm{Ag}^{+}$to $\mathrm{Ag}^{0}$ [32-34]. In the literature review, it was determined that the synthesis and application areas of silver nanoparticles based on plant extract were scattered over a wide spectrum, but there were limited studies in which silver nanoparticles were used in a shorter time and less amount. Lee, et al. investigated the catalytic activity of graphene oxide grafted gold nanoparticles and silver/silver chloride nanoparticles which prepared using green-synthesized by a Portulaca oleracea extract [35]. Huong, et al. observed the antibacterial activity of silver nanoparticles using Sapindus mukorossi fruit pericarp extract [36]. Karadirek, et al. preferred the ultrasound assisted green synthesis for silver nanoparticle attached activated carbon for levofloxacin adsorption [37]. Ahn, et al. reported the development of anticancer properties of silver nanoparticles which were prepared by plant extracts [38]. Albeladi, et al. prepared the silver nanoparticles using salvia officinalis leaf extract using the solution method to show its antimicrobial activity against staphylococcus aureus [25]. In this study, our scientific goal is to determine the effect of the surface tension and process parameters on the rheology characteristic of the synthesized SLE NPs. The attractive effect of our study is that, due to sonication-based cavitation, we have shown that the synthesized nanostructures, have a homogeneous distribution, can be obtained by using a low amount of silver solution for the reaction time of 5 $\min$.

\section{Materials and Methods}

\section{Materials}

The sage leaves (Salvia officinalis L.) were collected from Antalya (Turkey). Voucher specimen was stored in the Herbarium at the Faculty of Pharmacy, Hacettepe University, Turkey (HUEF 08014). The chemical composition of Sage leaves (Salvia officinalis L.) consisted of the polyphenolic compounds (phenolic acids, polyphenols, flavonoids, phenolic terpenes) and antioxidant constituents (carnosol, rosmadial, carnosine acid, rosmarinic acid and epirosmanol). Sodium hydroxide $(\mathrm{NaOH})$ was purchased from Merck Company. Silver nitrate $\left(\mathrm{AgNO}_{3}\right.$ ) (ACS reagent, $\geq 99.0 \%$, molecular weight: $\left.169.87 \mathrm{~g} \mathrm{~mol}^{-1}\right)$ purchased from Sigma Aldrich Company. We used the sterile syringe filters (Whatman 6900-2502 GD/X 25) which had a $25 \mathrm{~mm}$ filter, 0.22 -micron retention, and polyvinylidene difluoride (PVDF) membrane to prepare the nanostructure. Samples were stored in sterile polypropylene containers until use. The distilled water had a resistivity of $18.24 \mathrm{M} \Omega \mathrm{cm}$ at $25^{\circ} \mathrm{C}$. All chemicals and reagents were used without further purification. 


\section{Preparation of the sage leaves extract}

The fresh sage leaves were collected from Antalya (Turkey) in June and washed with distilled water for several times. They were dried in the vacuum oven until it reached a stable weight at $50{ }^{\circ} \mathrm{C} .10 \mathrm{~g}$ of the dried sage leaves were added into $450 \mathrm{ml}$ of distilled water for 7 days at room temperature in the dark medium without stirring. It was filtered using sterile syringe filters to obtain the SLE at room temperature and the yellow-colored extract was obtained. All samples were stored in sterile polypropylene containers until use at $25^{\circ} \mathrm{C}$.

\section{The dual sono/photochemical fabrication of the SLE-AgNPs}

The dual sono/photochemical synthesis route of SLE-AgNPs consisted of four steps. In the first step, $0.84 \mathrm{~g}$ of $\mathrm{AgNO}_{3}$ was dissolved in $500 \mathrm{ml}$ of distilled water and stirred for $10 \mathrm{~min}$ at room temperature by a magnetic stirrer. $0.4 \mathrm{~g}$ of $\mathrm{NaOH}$ was dissolved in $250 \mathrm{ml}$ of distilled water and stirred for $10 \mathrm{~min}$ at room temperature by a magnetic stirrer. In the second step, $100 \mathrm{ml}$ of the sage leaves extract was mixed with the $20 \mathrm{ml}$ of $\mathrm{AgNO}_{3}$ solution and sonicated for $5 \mathrm{~min}$ at $30 \%$ amplitude - frequency. In the third step, $2 \mathrm{ml}$ of $\mathrm{NaOH}$ solution was added slowly to this solution. By changing the color yellow to brown, the synthesis of SLE-AgNPs based was confirmed $(\mathrm{pH}=6)$ at $25^{\circ} \mathrm{C}$. Finally, SLE-AgNPs were filtered using sterile syringe filters and stored in sterile polypropylene containers until use at 25 ${ }^{\circ} \mathrm{C}$.

\section{Characterization}

We used different advanced techniques such as scanning electron microscopy (SEM) - energy-dispersive X-ray (EDX), transmission electron microscope (TEM), Fourier transform infrared (FTIR) spectroscopy, Brunauer-Emmett-Teller (BET), dynamic light scattering (DLS), and X-ray diffraction (XRD) to characterize the nanostructure. The SEM-EDX (FEI QUANTA, SEM 450) (TedPella, double-coated, $8 \mathrm{mmW} \times 20 \mathrm{~mL}$ ) was used to determine the morphology of the structure $(6-10 \mathrm{~mm}$ working distance, 0-130 Pa pressure, and voltage of 7-10 kV) under low vacuum medium. FTIR (Perkin Elmer, Spectrum Two) ( $\mathrm{KBr}$ powder and in the $4000-400 \mathrm{~cm}^{-1}$ frequency range with a resolution of $4 \mathrm{~cm}^{-1}$ and 8 scans) was used to observe the functional groups of the samples. Transmission electron microscope (TEM) (Hitachi HighTech HT7700) was used to visualize the nanostructure. BET (ASAP2020 model) was used to determine the specific surface area of the nanostructure. DLS measurements were performed using a Malvern Zetasizer Nano $\mathrm{ZS}^{\circ}$ instrument with 12 runs and were recorded at $25^{\circ} \mathrm{C}$. The surface tensions were performed using a force tensiometer (K11, KRUSS) under different experimental factors such as the amplitude, sonication time, and temperature.

\section{Designation of factors for the multiple-respon- sive optimization}

There are limited reports on the surface tensions of nanostructures in the literature. In this study, we focused on the design of the nanostructure for the multiple-responsive optimization using the rheological measurements of the SLE-AgNPs. These measurements based on the relationship between surface tensions of the samples with different experimental factors such as the amplitude (20-40\%), sonication time (1-5 $\mathrm{min}$ ) and temperature (25-45 ${ }^{\circ} \mathrm{C}$ ) were performed in three replicates.

\section{Error analysis}

In this study, we calculated different error analysis methods for SLE-AgNPs to evaluate the accuracy of the experimental results obtained the error analysis using surface tension results. These methods were the sum of squares of errors (SSE), the hybrid fractional error function (HYBRID), and the average relative error (ARE) all expressions and given in Table 1 (Equation 2.1-2.3) [39-41].

\section{Stability of the SLE-AgNPs over time}

The storage stability of Ag-PVP NPs was evaluated at times $0,8,12$, and 24 weeks using the UV-vis spectrophotometer and force tensiometer. Four sets of sterile centrifugal tubes containing SLE-AgNPs were prepared for each nominal size at $25^{\circ} \mathrm{C}$ and all samples were analyzed. The experimental measurements were performed in triplicate.

\section{Results and Discussion}

In this study, we investigated the morphology and functional groups of the synthesized SLE-AgNPs using different techniques such as SEM-EDX, TEM, FTIR, BET, DLS, and XRD. Also, we evaluated the effects of experimental factors such as the amplitude (20-40\%), sonication time (1-5 $\mathrm{min})$, and temperature $\left(25-45^{\circ} \mathrm{C}\right)$ on the rheological results. The experimental results presented the optimization of the novel nanostructure for the rheological 
Table 1: The formulas of the error methods.

\begin{tabular}{|l|l|l|l|}
\hline Expressions & Error analysis methods & Equation & Ref \\
\hline$\sum_{i=1}^{n}\left(\gamma_{c a l}-\gamma_{e}\right)_{i}^{2}$ & SSE & (2.1) & [39] \\
\hline$\frac{100}{n-p} \sum_{i=1}^{n}\left[\frac{\left(\gamma_{e}-\gamma_{c a l}\right)_{i}^{2}}{\gamma_{e}}\right]$ & HYBRID & (2.2) & [40] \\
\hline$\frac{100}{n-p} \sum_{i=1}^{n}\left|\frac{\gamma_{e}-\gamma_{c a l}}{\gamma_{e}}\right|_{i}$ & ARE & & \\
\hline
\end{tabular}

Where, $\mathrm{n}$ : Number of data points, p: Number of parameters, $\gamma_{e}$ : Experimental surface tension and $\gamma_{\text {cal }}$ : Calculated surface tension.

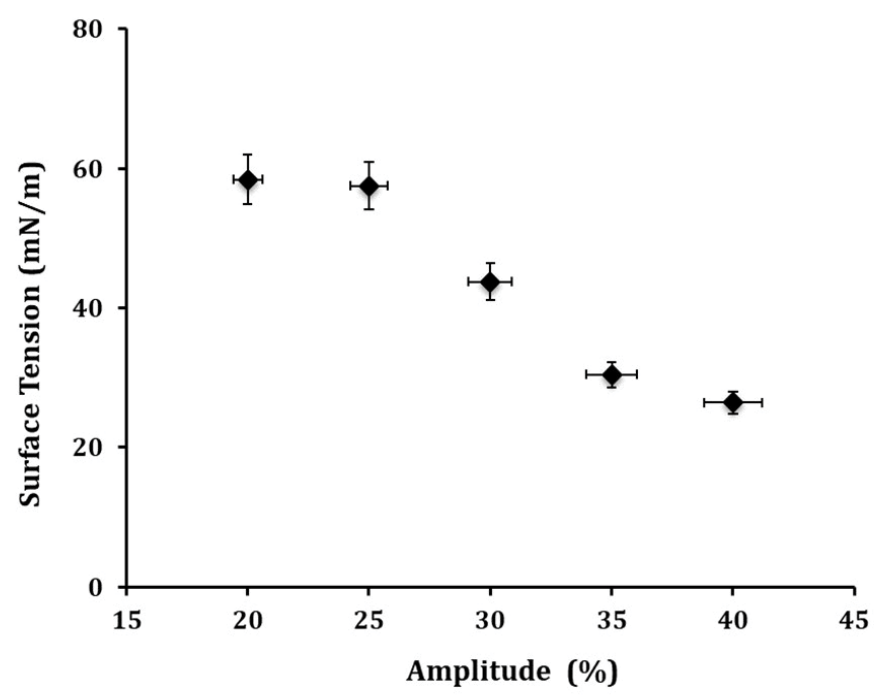

Figure 1: The effects of the amplitude (20-40\%) on the rheology of the SLE-AgNPs.

parameters with a focus on surface tensions.

\section{Rheological characterization of the SLE-AgNPs}

Rheological measurements have a major role in quality control during manufacturing, processing, and characterization of the structures $[42,43]$. As known, surface tension is an important criterion in the preparation of homogeneous formulations. We focused on the influences of experimental factors on the complex design which plays an important role in the fabrication of AgNPs with rheology measurements. We highlighted the applications of amplitude, sonication time and temperature as a rheological modifier on the surface tension. Also, we reported the optimization of ultrasonic conditions for the fabrication of SLE NPs. For this purpose, we compared experimentally the effects of the amplitude (20-40\%), sonication time (1-5 min), and temperature $\left(25-45^{\circ} \mathrm{C}\right)$ on the rheology of the SLE-AgNPs and these results were given in Figure 1, Figure 2 and Figure 3, respectively.

The surface tensions of SLE/solvent from 58.45 $\mathrm{mN} / \mathrm{m}$ to $26.43 \mathrm{mN} / \mathrm{m}$ with changing the amplitude of the process from $20-40 \%$ at $25{ }^{\circ} \mathrm{C}$ were recorded for SLE-AgNPs (Figure 1). According to the results, we observed that the surface tension of SLE/ solvent increased with the increase of the value of the amplitude. We proved that the surface tension changed at different amplitude values due to the intensity and frequency of the sonication. (Figure 1) [44].

The surface tensions of SLE/solvent from 42.67 $\mathrm{mN} / \mathrm{m}$ to $26.43 \mathrm{mN} / \mathrm{m}$ with changing concentration of the SLE-AgNPs from $10-0.1 \mathrm{mM}$ at $25{ }^{\circ} \mathrm{C}$ were recorded for $5 \mathrm{~min}$. The surface tensions of SLE/sol- 


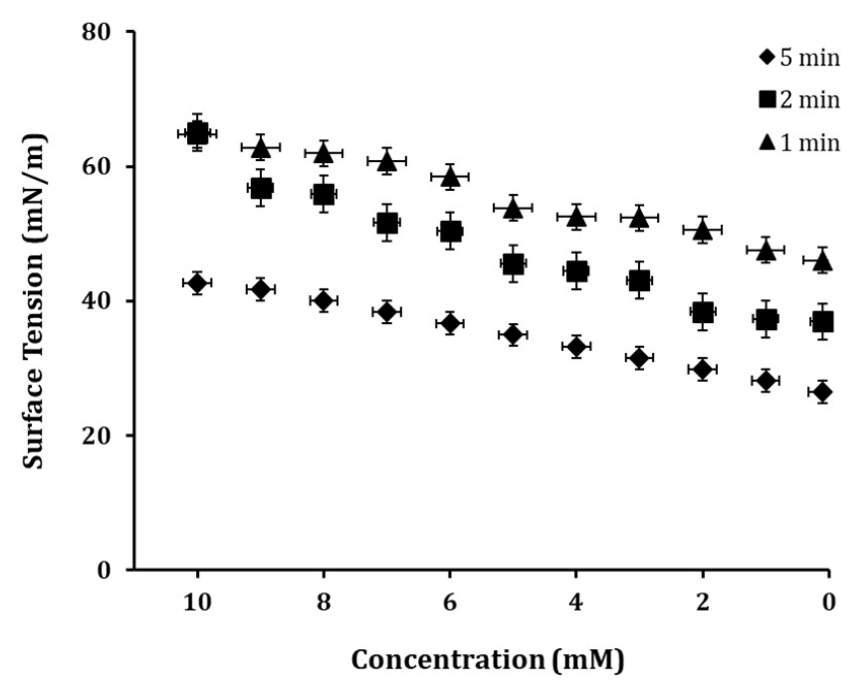

Figure 2: The effects of the sonication time (1-5 $\mathrm{min}$ ) on the rheology of the SLE-AgNPs.

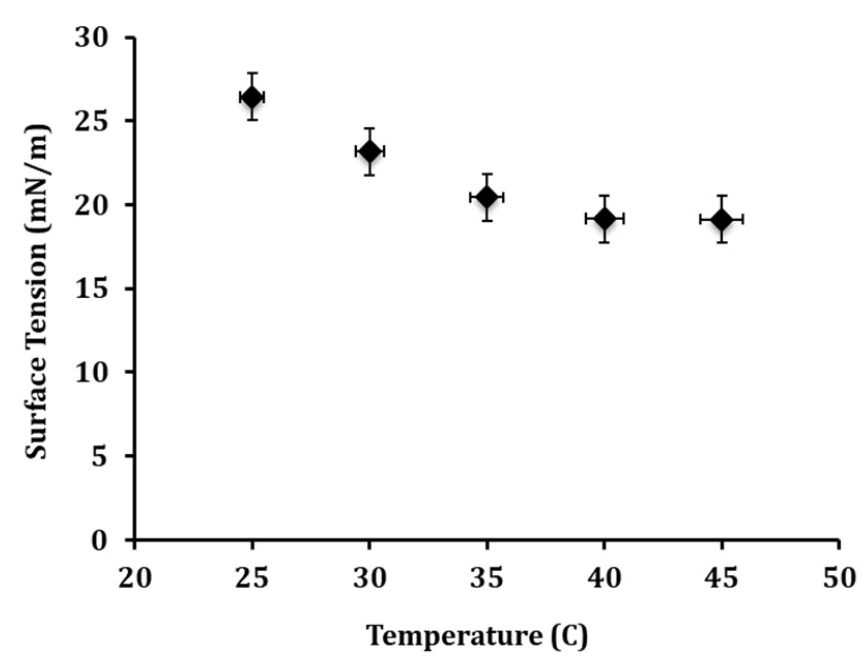

Figure 3: The effects of the temperature $\left(25-45^{\circ} \mathrm{C}\right)$ on the rheology of the SLE-AgNPs.

vent from $64.99 \mathrm{mN} / \mathrm{m}$ to $36.91 \mathrm{mN} / \mathrm{m}$ with changing concentration of the SLE-AgNPs from 10-0.1 $\mathrm{mM}$ at $25^{\circ} \mathrm{C}$ were recorded for $2 \mathrm{~min}$. The surface tensions of SLE/solvent from $64.77 \mathrm{mN} / \mathrm{m}$ to 46.04 $\mathrm{mN} / \mathrm{m}$ with changing concentration of the SLE-AgNPs from $10-0.1 \mathrm{mM}$ at $25{ }^{\circ} \mathrm{C}$ were recorded for $1 \mathrm{~min}$. According to the experimental results, we observed that the surface tensions of SLE/solvent increased with the increase of the concentration of SLE-AgNPs. It was proven that the surface tension changed at different sonication times depending on the AgNPs increased (Figure 2) $[45,46]$.

In Figure 3, the surface tensions of SLE/solvent from $26.43 \mathrm{mN} / \mathrm{m}$ to $19.12 \mathrm{mN} / \mathrm{m}$ with changing the temperature $\left(25-45^{\circ} \mathrm{C}\right)$ of the SLE-AgNPs were recorded. We observed that the surface tensions of SLE/solvent increased with the increase of the concentration of SLE-AgNPs. The effects of the amplitude, sonication time, and temperature on the surface tension of the nanostructure produced using the sonication method owing to the collapse of microbubbles in the nanostructure irradiated with ultrasound was experimentally examined in this study $[47,48]$. Accordingly, the sonochemical effects on the surface tension of the SLE mediated AgNPs owing to the ultrasound cavitation in the nanostructure were proven. To our knowledge, this study is the first to investigate the effects of the sonochemical method on the rheology with the SLE mediated AgNPs.

\section{Characterization of the SLE-AgNPs}

In this study, we presented the development of 


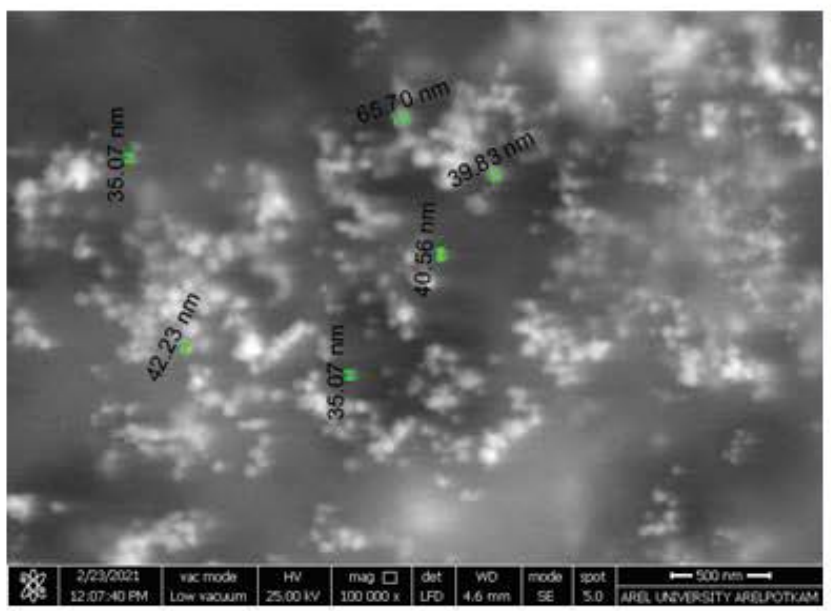

(a)

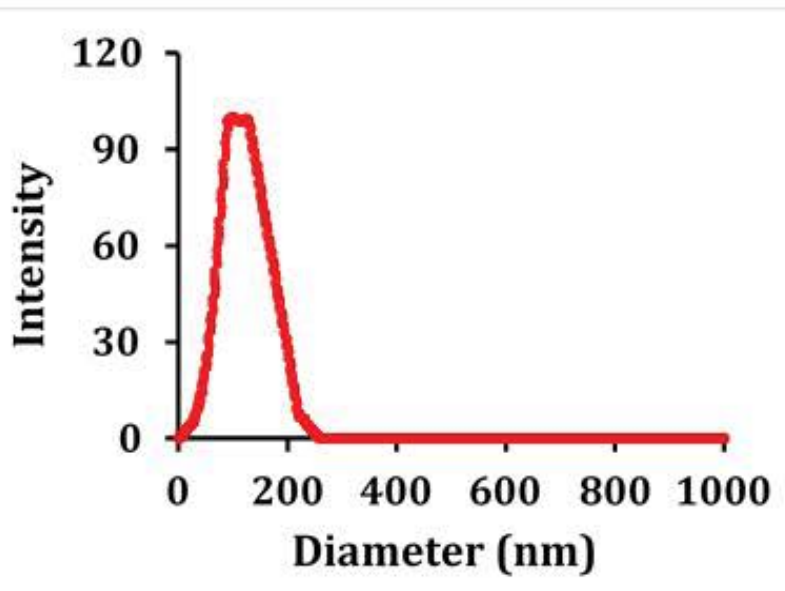

(c)

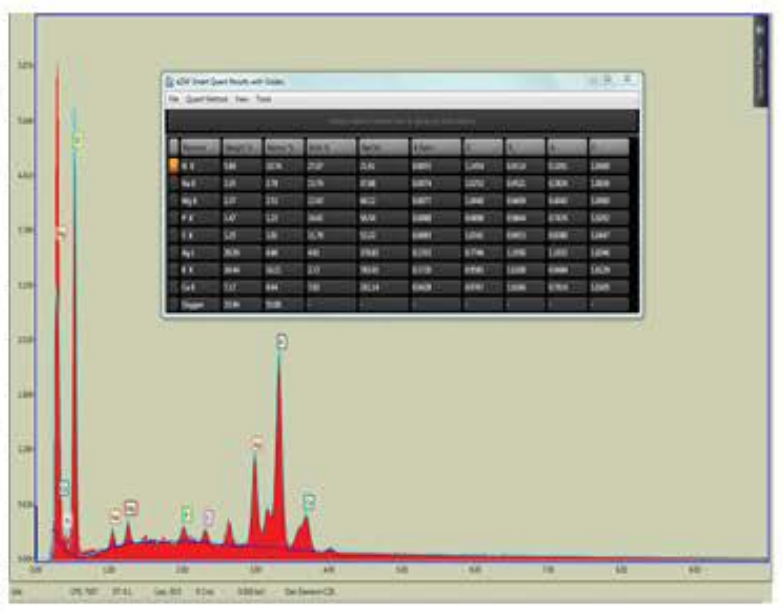

(b)

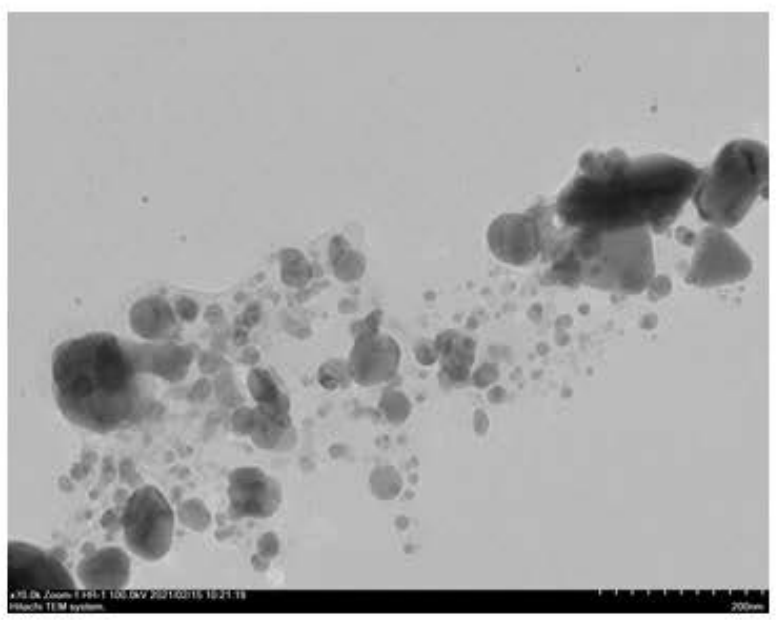

(d)

Figure 4: a) The SEM image; b) The EDX image; c) The TEM image; and d) DLS results of the SLE-AgNPs.

the dual sono/photochemical synthesis route using the SLE matrix as a phytochemical under the ultrasonic irradiations $\mathrm{Ag}^{+}$into AgNPs.

The surface morphology of synthesized SLE-AgNPs was performed by SEM and EDX techniques. (Figure $4 a$ and Figure $4 b$ ). The SEM analysis revealed that the SLE-AgNPs had spherical-shaped particles with a homogeneous distribution. The size of the nanoparticles varied between 35.07 and $65.70 \mathrm{~nm}$. Similar SEM results were obtained for the AgNPs prepared by Chowdhury, et al. [49]. The EDX analysis was performed to determine the elemental composition of the SLE-AgNPs. Figure $4 \mathrm{~b}$ illustrated the EDX spectrum of the SLE-AgNPs. The results indicated the presence of an intense peak due to the existence of $\mathrm{Ag}(20.20 \%), \mathrm{Ca}(7.17 \%)$, $\mathrm{O}$ (33.94\%), Na (3.35\%), Mg (2.37\%), P (1.47\%), S $(1.25 \%)$, and $\mathrm{K}(24.24 \%)$ elements in the biosynthe- sized SLE-AgNPs. The results of the DLS technique demonstrated that there was a uniform size distribution of the AgNPs with the hydrodynamic average size of $120 \mathrm{~nm}$ (Pdl: 0.007 and intercept: 0.944) and good dispersion of AgNPs in $10 \mathrm{mM}$ phosphate buffered saline (PBS) (1x pH 7.4) (Figure 4b). TEM image showed that biosynthesized AgNPs had a particle size distribution between 10 and $50 \mathrm{~nm}$ (Figure 4c). Thus, the results of the TEM analysis of the AgNPs we obtained were compatible with our SEM results. By using the results of BET measurements, we found that the surface area was 150.67 $\mathrm{m}^{2} / \mathrm{g}$.

The FTIR technique of SLE and SLE-AgNPs was used to investigate functional groups in the chemical structure and the interaction between AgNPs and SLE matrix in the region between 400 and 4000 $\mathrm{cm}^{-1}$. FTIR analysis of (a) SLE and (b) SLE-AgNPs 


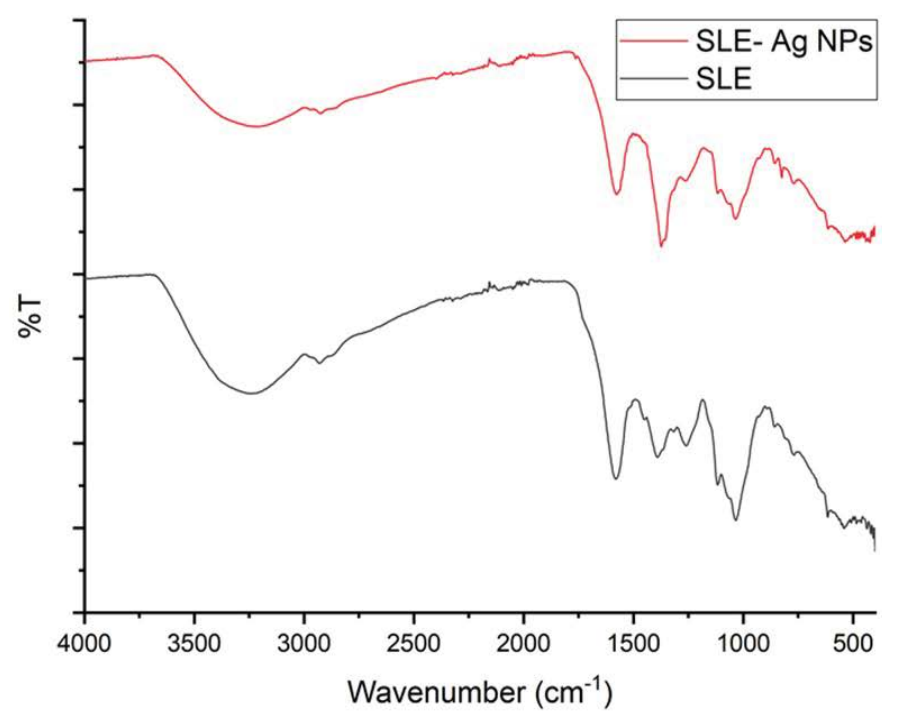

Figure 5: FTIR graphs of: a) SLE; and b) SLE-AgNPs.

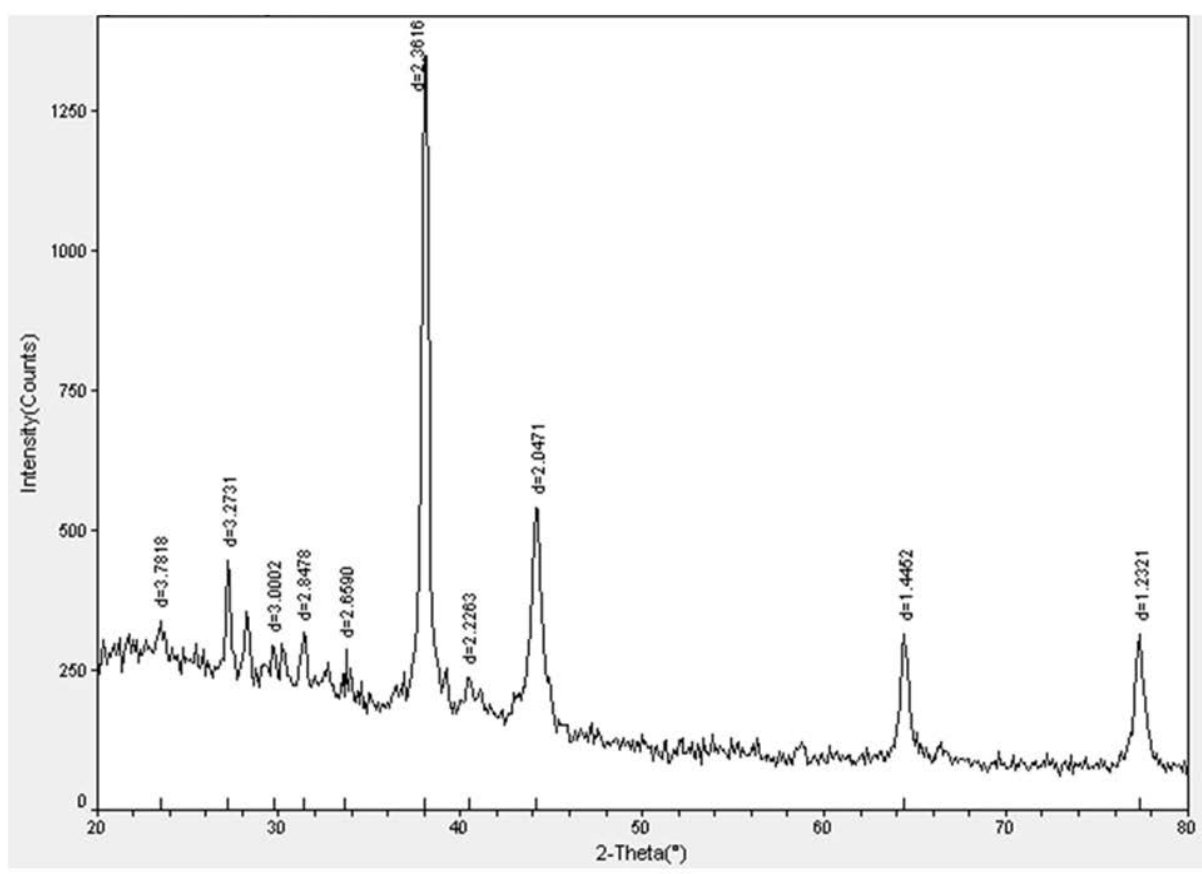

Figure 6: XRD result of the synthesized SLE-AgNPs.

were given in Figure 5. According to the FTIR results, the characteristic peaks of SLE were observed at $3270.68 \mathrm{~cm}^{-1}$ (-OH stretching vibration), 2927.41 $\mathrm{cm}^{-1}$ (C-H stretching vibration), $1585.19 \mathrm{~cm}^{-1}$ (C-O stretching vibrations), and $1031.73 \mathrm{~cm}^{-1}$ (C-O-C vibrations). Furthermore, the characteristic peaks of SLE-AgNPs were observed at $3303.46 \mathrm{~cm}^{-1}(-\mathrm{OH}$ stretching vibration), $1577.49 \mathrm{~cm}^{-1}(\mathrm{C}=\mathrm{O}$, carbon$\mathrm{yl}$ groups), $1373.07 \mathrm{~cm}^{-1}$ (C-H wagging vibrations) and $1035.59 \mathrm{~cm}^{-1}$ (amine groups $\mathrm{N}-\mathrm{H}$ ). According to these results, it was observed that the peaks of
$-\mathrm{OH}$ stretching vibration, $\mathrm{CO}$ stretching vibrations, and $\mathrm{C}-\mathrm{O}-\mathrm{C}$ vibrations were relatively smaller in the presence of AgNPs, but the relative intensity of the $\mathrm{C}-\mathrm{H}$ wagging vibrations peak increased. We also observed small peaks at $528 \mathrm{~cm}^{-1}$ due to the polyphenols which showed there are phenolic and carbonyl groups on the surface of the AgNPs. Similar spectra results indicate there was not much change in the SLE and SLE-AgNPs [29,36].

X-ray Diffraction (XRD) result of SLE-AgNPs was performed to investigate the effect of synthesized 
Table 2: The comparison of characterization results of the various AgNPs.

\begin{tabular}{|l|l|l|l|}
\hline Sample & Method & Characterization results & Ref. \\
\hline Plant extract - AgNPs & Biosynthesis & Spherical shape/17 nm & {$[52]$} \\
\hline Graphene oxide-AgNPs Hybrids & Sonochemical synthesis & Irregularly shaped/15-35 nm & {$[53]$} \\
\hline Perilla frutescens Leaf Extract- AgNPs & Phytosynthesis & Spherical shape/25.71 nm & {$[54]$} \\
\hline AgNPs in Y-zeolite substrate & Sonochemical synthesis & Spherical shape/10 nm & {$[55]$} \\
\hline Colloidal AgNPs & Sonochemical synthesis & Spherical shape/3.5 nm & {$[56]$} \\
\hline Purple heart plant leaves extract-AgNPs & Biosynthesis & Spherical shape/98 nm & {$[57]$} \\
\hline Myristica fragrans seed extract AgNPs & Biosynthesis & Spherical shape/5-20 nm & {$[58]$} \\
\hline Azadirachta indica leaf extract AgNPs & Biosynthesis & Spherical shape/4-19 nm & {$[59]$} \\
\hline Aegle marmelos fruit extract AgNPs & Biosynthesis & & {$[60]$} \\
\hline Allium ampeloprasum extract AgNPs & Biosynthesis & Spherical shape/116 nm & {$[61]$} \\
\hline SLE-AgNPs & Dual sono/photochemical & Spherical shape/17.56 nm & This study \\
\hline
\end{tabular}

AgNPs on the crystalline structure. The results were displayed in Figure 6. Figure 6 showed that characteristic peaks were formed around $2 \theta=38.1^{\circ}$, $44.4^{\circ}, 64.4^{\circ}$, and $77.4^{\circ}$ which corresponding to (111), (200), (220), and (311) planes of AgNPs, respectively (JCPDS file No.00-004-0783) [50,51]. We calculated the average crystallite size of SLE-AgNPs using Equation 3.1 (Debye-Scherer's equation) [51].

$$
D=0.9 . \lambda / \beta \cdot \cos \theta
$$

Where $\lambda: 0.1541 \mathrm{~nm}, \beta$ : The full width at half maximum (FWHM), $\theta$ : The Bragg's angle in degrees, and $D$ : The average particle size.

The average crystallite size of AgNPs was measured from the plane (1 111 ) using Eq. 3.1. It was calculated to be around $17.56 \mathrm{~nm}$ of SLE-AgNPs. According to our experimental results, the estimated fabrication mechanism of the AgNPs was described in Eqs. 3.2-3.9.

$$
\begin{aligned}
& \mathrm{H}_{2} \mathrm{O} \rightarrow{ }^{*} \mathrm{OH}+{ }^{*} \mathrm{H} \\
& { }^{*} \mathrm{OH}+{ }^{*} \mathrm{OH} \rightarrow \mathrm{H}_{2} \mathrm{O}_{2} \\
& { }^{*} \mathrm{H}+\mathrm{O}_{2} \rightarrow \mathrm{HO}_{2}^{*} \\
& \mathrm{HO}^{-}+\mathrm{H}_{2} \mathrm{O}_{2} \leftrightarrow \mathrm{HOO}^{-}+\mathrm{H}_{2} \mathrm{O} \\
& \mathrm{HO}_{2}{ }^{*}+{ }^{*} \mathrm{OH} \rightarrow \mathrm{H}_{2} \mathrm{O}+\mathrm{O}_{2} \\
& \mathrm{AgNO}_{3} \rightarrow \mathrm{Ag}^{+}+\mathrm{NO}_{3}^{-}
\end{aligned}
$$

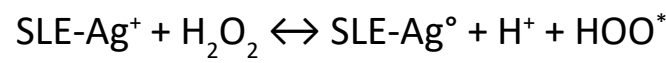

$$
\begin{aligned}
& \mathrm{HOO}^{-}+(\mathrm{SLE}-) \mathrm{Ag}^{+} \leftrightarrow \mathrm{HOO}^{*}+\mathrm{SLE}-\mathrm{Ag}^{\circ}
\end{aligned}
$$

In previous studies, it was proven that bio-based products such as nanostructures obtained from biological systems and products mediated by the vegetable, animal oils, and plant extracts are bio- degradable. For this purpose, our scientific goal was to prepare the biodegradable plant extract mediated silver nanoparticles without using any toxic reducing agents in this study. The results of the synthesized AgNPs were appreciable from the comparison with the results of previous studies in the literature (Table 2) [52-61].

\section{Error analysis of SLE-AgNPs}

In this study, the error analysis results of SLE-AgNPs were compared with different error methods such as SSE, HYBRID, and ARE using the surface tension measurements. When Eq. 2.1-2.3 were applied with the repetition of the data obtained from the surface tension measurements, the minimum error results were observed. According to results, we calculated the parameters of SSE, HYBRID, and ARE were $0.12,0.15$, and 0.48 , respectively. Accordingly, it was proven with the minimum error results. Although such error calculations were reported in the literature, no study gives the relation of the surface tension for the AgNPs. Also, there are no systematic reports on the comparison of the error methods of the AgNPs in the literature to determine the change in the surface tension of the nanostructure under the sonochemical cavitation.

\section{Stability of SLE-AgNPs}

The storage stability of the SLE-AgNPS was evaluated using surface tension measurements and UV-Vis spectroscopy. The storage stability of the SLE-AgNPS was monitored by checking its surface tension and absorbance at $25^{\circ} \mathrm{C}$. Also, the storage stability of the SLE-AgNPs was confirmed by the UV-Vis absorption spectra measured as a func- 


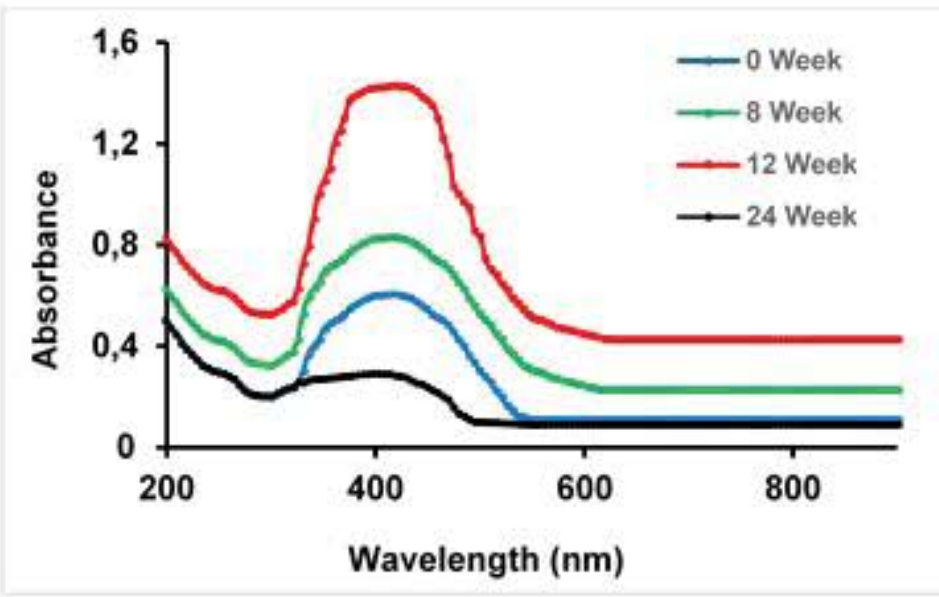

(a)

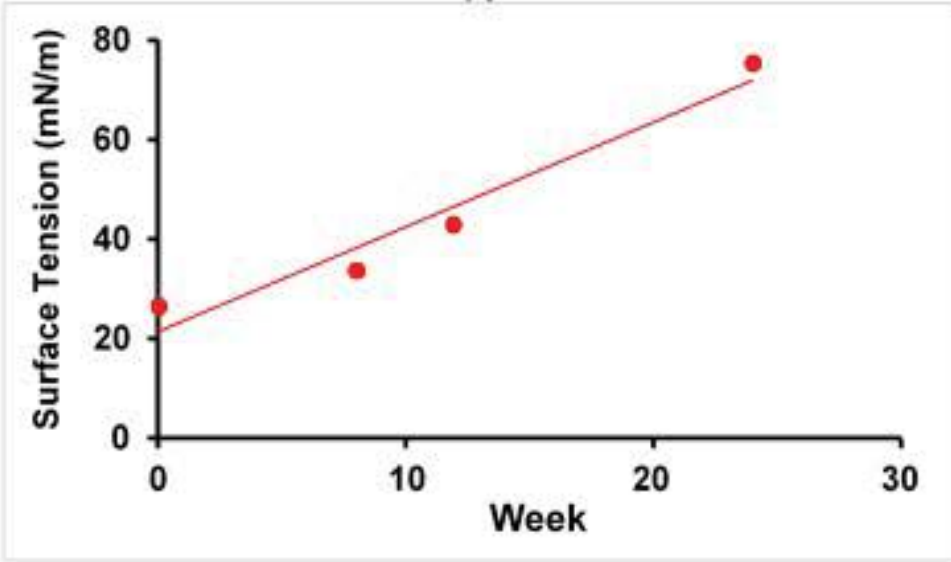

(b)
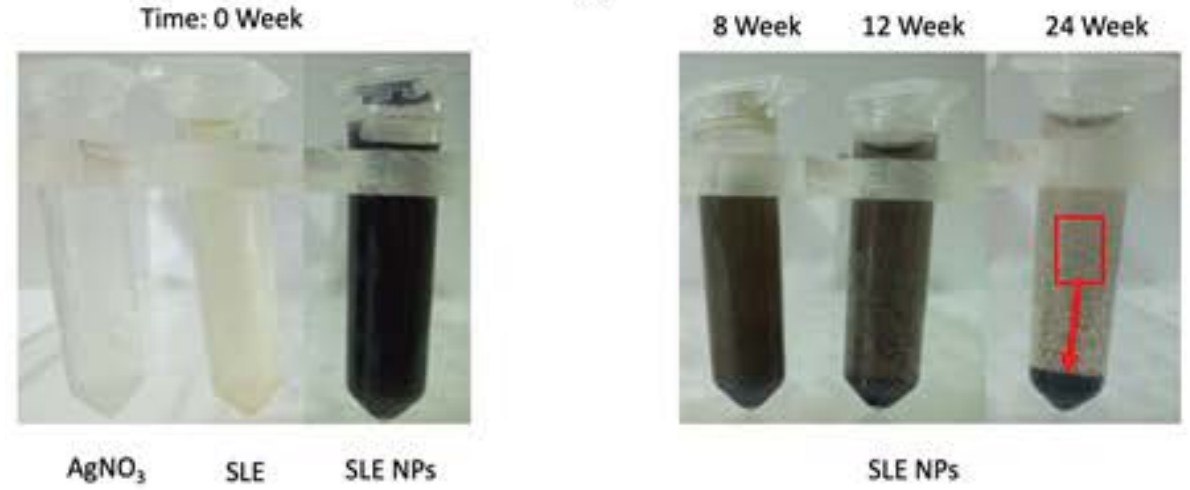

Figure 7: a) The UV-Vis spectra; b) Surface tension of the SLE-AgNPs; and c) The photographs of the silver nitrate solution, SLE, and SLE-AgNPs at different times.

tion of time (0-24 Week). Besides, SLE-AgNPs were proved to have two characteristic peaks at absorption spectra 265 and $465 \mathrm{~nm}$ (Figure 7). Furthermore, the surface tension of the SLE-AgNPS after 24 weeks of storage was found to be $75.43 \pm 1.0$ $\mathrm{mN} / \mathrm{m}$ (Figure 7). These results exhibited an indication of its excellent stability with its photographs at different times for the SLE NPs [62].

\section{Conclusion}

In summary, the SLE-AgNPs were prepared successfully by the green dual sono/photochemical synthesis method and the synthesized SLE-AgNPs displayed sensitivity to factors such as the amplitude (20-40\%), sonication time (1-5 $\mathrm{min})$, and temperature $\left(25-45^{\circ} \mathrm{C}\right)$ on the surface tension. The effective experimental factors were calculated to 
determine the optimum condition of the preparation of the uniform nanostructure with compatible error results. The characterization results showed that the SLE-AgNPs had a uniform spherical-shaped structure with a large surface area $\left(150.67 \mathrm{~m}^{2} / \mathrm{g}\right)$ and have potential in various applications of scientific and industrial fields in the future.

\section{Declaration of Competing Interest}

The authors declare no conflict of interests.

\section{References}

1. Prabha S, Durgalakshmi D, Rajendran S, Lichtfouse E (2020) Plant-derived silica nanoparticles and composites for biosensors, bioimaging, drug delivery and supercapacitors: A review. Environmental Chemistry Letters 2020: 1-25.

2. Gupta DSK (2020) Study of nanotechnology and its application. J Phy Opt Sci 2: 1-7.

3. Wang Z, Zou Y, Li Y, Cheng Y (2020) Metal-containing polydopamine nanomaterials: Catalysis, energy, and theranostics. Small 16: 1907042.

4. Saleh TA (2020) Nanomaterials: Classification, properties, and environmental toxicities. Environmental Technology \& Innovation 20: 101067.

5. Holec D, Dumitraschkewitz $P$, Vollath $D$, Fischer FD (2020) Surface energy of Au nanoparticles depending on their size and shape. Nanomaterials 10: 484.

6. Luo YH, Chang LW, Lin P (2015) Metal-based nanoparticles and the immune system: Activation, inflammation, and potential applications. BioMed Research International 2015: 143720.

7. Makvandi $P$, Wang $C Y$, Zare EN, Borzacchiello A, Niu LN, et al. (2020) Metal-based nanomaterials in biomedical applications: Antimicrobial activity and cytotoxicity aspects. Advanced Functional Materials 30: 1910021.

8. Yaqoob SB, Adnan R, Khan RMR, Rashid M (2020) Gold, silver, and palladium nanoparticles: A chemical tool for biomedical applications. Frontiers in chemistry 8: 376.

9. Singh J, Dutta T, Kim KH, Rawat M, Samddar $P$, et al. (2018) 'Green'synthesis of metals and their oxide nanoparticles: Applications for environmental remediation. Journal of Nanobiotechnology 16: 84.

10.Annu AA, Ahmed S (2018) Green synthesis of metal, metal oxide nanoparticles, and their various applications. Handbook of Ecomaterials 2018: 1-45.

11.Ahmed KBR, Nagy AM, Brown RP, Zhang Q, Malghan
SG, et al. (2017) Silver nanoparticles: Significance of physicochemical properties and assay interference on the interpretation of in vitro cytotoxicity studies. Toxicology in Vitro 38: 179-192.

12.Xu L, Wang YY, Huang J, Chen CY, Wang ZX, et al. (2020) Silver nanoparticles: Synthesis, medical applications and biosafety. Theranostics 10: 8996-9031.

13. Vysotsky VV, Dmitriev AS, Mikhailova IA, Chernyshova KF, Souvorova OV, et al. (2020) Graphene nanofl akes and hybrid nanocomposites with gold and silver nanoparticles: Optical and thermal properties. Russian Chemical Bulletin 69: 32-42.

14. Mohamed N, Madian NG (2020) Evaluation of the mechanical, physical and antimicrobial properties of chitosan thin films doped with greenly synthesized silver nanoparticles. Materials Today Communications 25: 101372.

15. Kumar A, Behl T, Chadha S (2020) Synthesis of physically crosslinked PVA/Chitosan loaded silver nanoparticles hydrogels with tunable mechanical properties and antibacterial effects. International Journal of $\mathrm{Bi}$ ological Macromolecules 149: 1262-1274.

16.Sreeram KJ, Nidhin M, Nair BU (2008) Microwave assisted template synthesis of silver nanoparticles. Bulletin of Materials Science 31: 937-942.

17.Tsujia T, Thang DH, Okazaki Y, Nakanishi M, Tsuboi $Y$, et al. (2008) Preparation of silver nanoparticles by laser ablation in polyvinylpyrrolidone solutions. Applied Surface Science 254: 5224-5230.

18. Tippayawat $P$, Phromviyo N, Boueroy $P$, Chompoosor A (2016) Green synthesis of silver nanoparticles in aloe vera plant extract prepared by a hydrothermal method and their synergistic antibacterial activity. Peer J 4: e2589.

19. Khayati G, Janghorban K (2012) The nanostructure evolution of $\mathrm{Ag}$ powder synthesized by high energy ball milling. Advanced Powder Technology 23: 393397.

20. Wang H, Qiao X, Chen J, Ding S (2005) Preparation of silver nanoparticles by chemical reduction method. Colloids and Surfaces A: Physicochemical and Engineering Aspects 256: 111-115.

21.Rodriguez-Sanchez L, Blanco $M$, Lopez-Quintela $M$ (2000) Electrochemical synthesis of silver nanoparticles. The Journal of Physical Chemistry B 104: 96839688 .

22. Naik RR, Stringer SJ, Agarwal G, Jones SE, Stone MO (2002) Biomimetic synthesis and patterning of silver nanoparticles. Nature Materials 1: 169-172. 
23.Sarina S, Waclawik ER, Zhu H (2013) Photocatalysis on supported gold and silver nanoparticles under ultraviolet and visible light irradiation. Green Chemistry 15: 1814-1833.

24.Wang F, Nie N, He H, Tang Z, Chen Z, et al. (2017) UItrasonic-assisted sintering of silver nanoparticles for flexible electronics. The Journal of Physical Chemistry C 121: 28515-28519.

25.Albeladi SSR, Malik MA, Al-thabaiti SA (2020) Facile biofabrication of silver nanoparticles using Salvia officinalis leaf extract and its catalytic activity towards Congo red dye degradation. Journal of Materials Research and Technology 9: 10031-10044.

26.Yong BC, Raman AAA, Buthiyappan A, Abidin MIIZ (2020) Synthesis and characterization of sugarcane bagasse cellulose-capped silver nanoparticle using ultrasonic irradiation for the adsorption of heavy metal. Asia-Pacific Journal of Chemical Engineering 15: e2433.

27.Alhag SK, Al-Mekhlafi FA, Abutaha N, Galil FMAA, Wadaan MA (2021) Larvicidal potential of gold and silver nanoparticles synthesized using Acalypha fruticosa leaf extracts against Culex pipiens (Culicidae: Diptera). Journal of Asia-Pacific Entomology 24: 184189.

28.Lekeshmanaswamy M, Anusiyadevi K (2020) Biosynthesis of silver nanoparticles using Pergularia daemia (Hamilton, 1822) leaf extract and its enhanced antibacterial activity against gram negative bacteria (Escherichia coli). Materials Today: Proceedings.

29.Manik UP, Nande A, Raut S, Dhoble SJ (2020) Green synthesis of silver nanoparticles using plant leaf extraction of artocarpus heterophylus and azadirachta indica. Results in Materials 6: 100086.

30.Yugay YA, Usoltseva RV, Silant'ev VE, Egorova AE, Karabtsov AA, et al. (2020) Synthesis of bioactive silver nanoparticles using alginate, fucoidan and laminaran from brown algae as a reducing and stabilizing agent. Carbohydrate Polymers 245: 116547.

31.Chandra A, Bhattarai A, Yadav AK, Adhikari J, Singh $M$, et al. (2020) Green synthesis of silver nanoparticles using tea leaves from three different elevations. Chemistry Select 16: 4239-4246.

32.Naz S, Shams F, Tabassum S, Ul-Haq I, Ashraf M, et al. (2017) Kinnow peel extract as a reducing and capping agent for the fabrication of silver NPs and their biological applications. IET nanobiotechnology 11: 1040-1045.

33. Mohamad NAN, Arham NA, Jai J, Hadi A (2014) Plant extract as reducing agent in synthesis of metallic nanoparticles: A review. Advanced Materials Research 832: 350-355.

34.Ahmed S, Saifullah, Ahmad M, Swami BL, Ikram S (2016) Green synthesis of silver nanoparticles using Azadirachta indica aqueous leaf extract. Journal of Radiation Research and Applied Sciences 9: 1-7.

35.Lee YJ, Park Y (2020) Graphene oxide grafted gold nanoparticles and silver/silver chloride nanoparticles green-synthesized by a Portulaca oleracea extract: Assessment of catalytic activity. Colloids and Surfaces A: Physicochemical and Engineering Aspects 607: 125527.

36. Huong VTL, Nguyen NT (2020) Green synthesis, characterization and antibacterial activity of silver nanoparticles using Sapindus mukorossi fruit pericarp extract. Materials Today: Proceedings.

37. Karadirek Ş, Okkay H (2019) Ultrasound assisted green synthesis of silver nanoparticle attached activated carbon for levofloxacin adsorption. Journal of the Taiwan Institute of Chemical Engineers 105: 3949.

38. Ahn EY, Park Y (2020) Anticancer prospects of silver nanoparticles green-synthesized by plant extracts. Materials Science and Engineering: C 116: 111253.

39.Gan S, Zakaria S, Jaafar SNS (2019) Enhanced mechanical properties of hydrothermal carbamated cellulose nanocomposite film reinforced with graphene oxide. Carbohydrate Polymers 172: 284-293.

40.Das B, Chattopadhyay P, Upadhyay A, Gupta K, Mandal $M$, et al. (2014) Biophysico-chemical interfacial attributes of $\mathrm{Fe} 3 \mathrm{O} 4$ decorated MWCNT nanohybrid/ bio-based hyperbranched polyurethane nanocomposite: An antibacterial wound healing material with controlled drug release potential. New Journal of Chemistry 38: 4300-4311.

41. Mahdavinia GR, Massoudi A, Baghban A, Shokri E (2014) Study of adsorption of cationic dye on magnetic kappa-carrageenan/PVA nanocomposite hydrogels. Journal of Environmental Chemical Engineering 2: 1578-1587.

42.Benezech T, Maingonnat JF (1994) Characterization of the rheological properties of yoghurt-A review. Journal of Food Engineering 21: 447-472.

43.Cai Z, Wu J, Wu M, Li R, Wang P, et al. (2020) Rheological characterization of novel carboxymethylated curdlan-silica hybrid hydrogels with tunable mechanical properties. Carbohydrate Polymers 230: 115578.

44.Cabrera-Trujillo MA, Sotelo-Díaz LI, Quintanilla-Carvajal MX (2016) Effect of amplitude and pulse in low 
frequency ultrasound on oil/water emulsions. DYNA 83: 63-68.

45. Hernandez C, Nieves L, de Leon Al C, Advincula R, Exner AA (2018) Role of surface tension in gas nanobubble stability under ultrasound. ACS Applied Materials \& Interfaces 10: 9949-9956.

46.Oroian M (2013) Measurement, prediction and correlation of density, viscosity, surface tension and ultrasonic velocity of different honey types at different temperatures. Journal of Food Engineering 119: 167172.

47.Rae J, Ashokkumar M, Eulaerts O, Sonntag C, Reisse $\mathrm{J}$, et al. (2005) Estimation of ultrasound induced cavitation bubble temperatures in aqueous solutions. Ultrasonics Sonochemistry 12: 325-329.

48. Elsupikhe RF, Elsupikhe, Shameli K, Ahmad MB, Ibrahim NA, et al. (2015) Green sonochemical synthesis of silver nanoparticles at varying concentrations of K-carrageenan. Nanoscale Research Letters 10: 916.

49. Chowdhury RA, Dhar SA, Das S, Nahian Md. K, Qadirb Md. R (2020) Green synthesis and characterization of silver nanoparticles from the aqueous extract of the leaves of Citrus aurantifolia. Materials Today: Proceedings.

50. Harisha KS, Parushuram N, Ranjana R, Martis LJ, Narayana B, et al. (2020) Characterization and antibacterial properties of biogenic spherical silver nanoparticles. Materials Today: Proceedings.

51.Mohan S, Panneerselvam K (2021) An investigation on antibacterial filler property of silver nanoparticles generated from Walnut shell powder by insitu process. Materials Today: Proceedings 39: 368-372.

52.Salih TA, Hassan KT, Majeed SR, Ibraheem IJ, Hassan OM, et al. (2020) In vitro scolicidal activity of synthesised silver nanoparticles from aqueous plant extract against echinococcus granulosus. Biotechnology Reports 28: e00545.

53.Rodríguez-Otamendi DI, Meza-Laguna V, Acosta D, Álvarez-Zauco E, Huerta L, et al. (2021) Eco-friendly synthesis of graphene oxide-silver nanoparticles hybrids: The effect of amine derivatization. Diamond and Related Materials 111: 108208.

54.Reddy NV, Li H, Hou T, Bethu MS, Ren Z, et al. (2021) Phytosynthesis of silver nanoparticles using perilla frutescens leaf extract: Characterization and evaluation of antibacterial, antioxidant, and anticancer activities. International Journal of Nanomedicine 16: 15-29.

55. Talebi J, Halladj R, Askari S (2010) Sonochemical synthesis of silver nanoparticles in Y-zeolite substrate. Journal of materials science 45: 3318-3324.

56.Darroudi M, Zak AK, Muhamad MR, Huang NM, Hakimi M (2012) Green synthesis of colloidal silver nanoparticles by sonochemical method. Materials Letters 66: 117-120.

57. Hasnain MS, Javed Md. N, Alam Md. S, Rishishwar P, Rishishwar S, et al. (2019) Purple heart plant leaves extract-mediated silver nanoparticle synthesis: Optimization by Box-Behnken design. Materials Science and Engineering: C 99: 1105-1114.

58.Sharma G, Asharma AR, Kurian M, Bhavesh R, Nam JS (2014) Green synthesis of silver nanoparticle using myristica fragrans (nutmeg) seed extract and its biological activity. Digest Journal of Nanomaterials \& Biostructures 9: 325-332.

59. Nazeruddin GM, Prasad NR, Waghmare SR, Garadkar KM, Mulla IS (2014) Extracellular biosynthesis of silver nanoparticle using Azadirachta indica leaf extract and its anti-microbial activity. Journal of Alloys and Compounds 583: 272-277.

60.Patil S, Muthusamy P (2020) A bio-inspired approach of formulation and evaluation of Aegle marmelos fruit extract mediated silver nanoparticle gel and comparison of its antibacterial activity with antiseptic cream. European Journal of Integrative Medicine 33: 101025.

61.Jalilian F, Chahardoli A, Sadrjavadi K, Fattahi A, Shokoohinia Y (2020) Green synthesized silver nanoparticle from Allium ampeloprasum aqueous extract: Characterization, antioxidant activities, antibacterial and cytotoxicity effects. Advanced Powder Technology 31: 1323-1332.

62. Dhanya CS, Paul W, Victor SP, Joseph R (2021) On improving the physiological stability of curcuminoids: Curcumininoid-silver nanoparticle complex as a better and efficient therapeutic agent. Nano-Structures \& Nano-Objects 25: 100661. 